\title{
Cross-Layer Network Survivability Under Multiple Cross-Layer Metrics
}

\author{
Zhili Zhou, Member, IEEE, Tachun Lin, Member, IEEE, \\ Krishnaiyan Thulasiraman, Fellow, IEEE,Guoliang Xue, Fellow, IEEE, and Sartaj Sahni, Fellow, IEEE
}

\begin{abstract}
Given a cross-layer network with logical and physical topologies, the survivable logical topology routing problem is to route each link in the logical layer with a path in the physical topology between the end nodes of a logical link such that the logical topology remains connected after a physical link fails. The Mixed-Integer Linear Programming (MILP) formulation to determine such a routing has been considered in a recent paper [1]. Using this formulation as a basic building block, in this paper we present unified MILP formulations to determine a survivable logical topology routing that also satisfies one of four cross-layer metrics: (1) minimizing the number of logical links to be added to guarantee the existence of survivable logical topology routing; (2) maximizing the capacity of the logical topology; (3) maximizing the connectivity of the logical topology after a physical link failure; and (4) maximizing the minimum crosslayer cut. We also provide heuristics for these problems and compare the performance of these heuristics and MILPs using extensive simulations.
\end{abstract}

Index Terms-Cross-layer networks, cross-layer survivability, network augmentation, cross-layer reliability metrics, IP-overWDM optical networks, network virtualization, mathematical programming.

\section{INTRODUCTION}

$\mathbf{O}$ VER the last decade, an explosive growth in mobile and Internet traffic has pushed demands for higher capacity in data transmission of telecommunication networks. The data transmission rate of fiber optics networks has reached 305 $\mathrm{Tb} / \mathrm{s}$ according to a recent literature [2]. When failure occurs under large-capacity transmission, it causes broader impacts and catastrophic results. Thus, reliability has become an issue of great interest in the design of modern telecommunication networks, and has been intensively studied in single-layer communication networks.

In this paper, we focus on assessing cross-layer network reliability. An example of a cross-layer network architecture is Internet Protocol over Wavelength Division Multiplexing (IPover-WDM) networks, which is composed of logical (upperlayer, IP) and physical (lower-layer, WDM) networks. The demands of a link in the logical network are transmitted through a path connecting the corresponding node pair in the

Zhili Zhou is with IBM Research Collaboratory, Singapore. (e-mail: zhili@sg.ibm.com.)

Tachun Lin is with the Department of Computer Science and Information Systems, Bradley University, Peoria, IL 61625, USA.

Krishnaiyan Thulasiraman is with the School of Computer Science, University of Oklahoma, Norman, OK 73019, USA

Guoliang Xue is with the School of Computing, Informatics, and Decision Systems Engineering, Arizona State University, Tempe, AZ 85287, USA.

Sartaj Sahni is with Computer and Information Science and Engineering Department, University of Florida, Gainesville, FL 32611, USA. physical network. This logical-link to physical-path mapping is called cross-layer mapping. We will use logical, upper-layer, and IP networks interchangeably, as well as physical, lowerlayer, and WDM networks. We also refer to the topologies of the IP and WDM networks as logical and physical topologies, respectively.

In a logical topology, nodes and links represent IP routers and links connecting them, respectively. Similarly, physical nodes represent optical cross-connect (OXC) and optical adddrop multiplexer (OADM), while physical edges connecting them represent optical fibers. A lightpath is a cross-layer mapping of a logical link onto a path connecting corresponding physical nodes, through which transmission occurs on a single wavelength thereby bypassing opto-electro-optic (O-EO) conversions on the intermediate nodes of the path.

Another example of cross-layer networks is encountered in the study of network virtualization, where the virtual and real (physically existing) networks are, respectively, the logical and physical networks. Under the assumption that each virtual node is mapped to a physical node, the implementation of virtual link is also realized through the cross-layer mapping.

Due to the fact that each physical link may carry traffic/demands of multiple logical links, a single physical link failure could disconnect multiple logical links in cross-layer networks. This has given rise to an extensive interest in the study of reliability issues in cross-layer networks under multiple survivability evaluation metrics. A general definition of cross-layer network survivability is to identify a cross-layer mapping so that the logical network remains connected after any single physical link failure. A mapping which satisfies this evaluation metric is called a survivable cross-layer mapping. This survivability study is applicable to any cross-layer networks. It is obvious that survivability of a mapping can be guaranteed if the lightpaths corresponding to this mapping are all link-disjoint. However, this is only a sufficient condition.

Furthermore, with the consideration of logical link demands and physical link capacities, the evaluation metric for crosslayer mapping has been extended from pure survivability metric to an integrated one satisfying survivability and demands. This extension metric was first introduced by Lin et al. [1][3]. A cross-layer mapping is called a weakly survivable mapping if the logical network remains connected after a single physical link failure. A weakly survivable routing that also satisfies physical link capacity and logical demand constraints is called strongly survivable. Figure 1 illustrates weakly and strongly survivable cross-layer mappings. Here the vertical dotted lines show the correspondence between logical and 


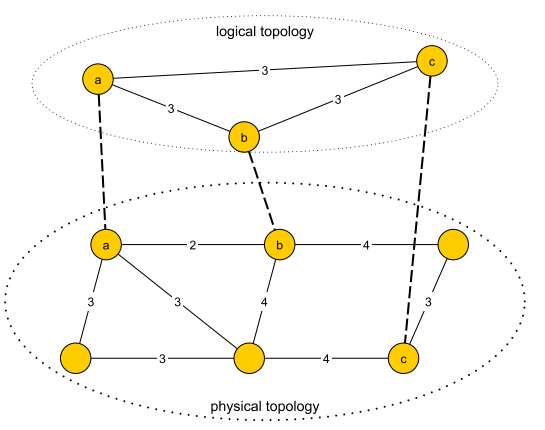

(a) Given physical/logical topologies

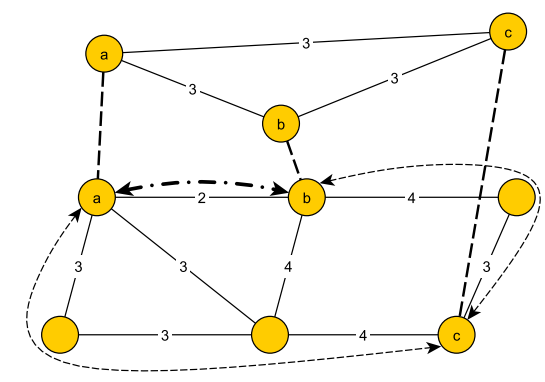

(b) Weakly survivable mapping

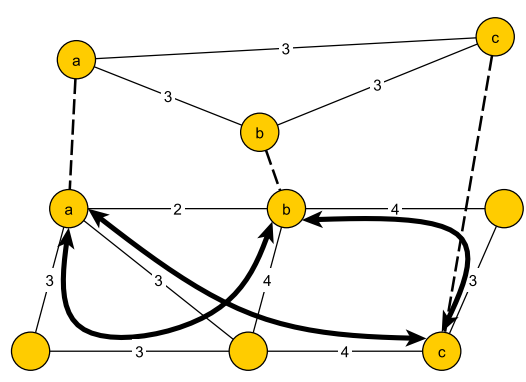

(c) Strongly survivable mapping

Fig. 1. Cross-layer Survivability Illustration

physical nodes. Also, the capacities of physical links are shown on the corresponding links. Figure 1(a) provides topologies and attributes of logical and physical networks. Figures 1(b) and 1(c) illustrate survivable cross-layer mappings with partial and full demand satisfaction, namely weakly and strongly survivable mapping, correspondingly.

Most of the papers in the literature deal with determining a survivable routing of logical links. In contrast, our focus in this paper is to study integrated design of cross-layer mappings that satisfy certain quality of service requirements besides the survivability condition. For this purpose we identify three cross-layer metrics and present MILPs to determine a routing that maximizes one of these metrics. We also provide an MILP for the logical topology augmentation problem. Our MILPs start with an MILP for the survivable logical topology routing problem. We have chosen the MILP given in [1] which enforces connectivity requirement through the existence of a spanning tree and does not require exponential number of variables used by other ILPs. This MILP is reviewed in Section III after a survey of literature on related works in Section II. All the MILPs developed in this paper use this MILP as a building block. The main contributions of the paper are summarized below.

- Minimum Logical Topology Augmentation Problem: Given a logical topology, the augmentation problem is to add a minimum number of additional links to the logical topology that guarantees the existence of a survivable routing of all the logical links of the augmented logical graph. Two earlier works that considered this problem are Thulasiraman et al. [4] and Liu and Ruan [5]. Neither of these two approaches solves the minimum augmentation problem. In Section IV we give an MILP that solves the minimum augmentation problem.

- Introduction of a new metric "after-failure connectivity" and determining a routing that maximizes after-failure connectivity: Suppose a logical graph is $k$-connected (That is, at least $k$ logical links have to be disconnected to disconnect the logical graph). A survivable routing only guarantees that the logical graph will remain 1-connected after a physical link failure. Since one physical link failure may disconnect several logical links, a number of links (more than $k$ links) may get disconnected when two or more failures occur, causing disconnection of the logical graph. To achieve a routing that can survive more than one physical link failure, we need to ensure that the connectivity of the logical graph is as high as possible after a single physical failure. With this in view in Section V-A we define a new metric called after-failure connectivity of the logical graph that is the connectivity of the logical graph after a single physical failure. The larger the value of after-failure connectivity of a routing the better will be its ability to survive multiple physical faults. Thus the after-failure connectivity of a routing is a measure of the ability of the logical graph to remain connected after multiple physical link failures. Our interest is to determine a routing that has as high a value of after-failure connectivity as possible. We give in Section V-A an MILP to determine a survivable routing that maximizes the after-failure connectivity of the logical graph. Our MILP has two significant features: 1) Normally calculating the connectivity of the logical graph after each physical failure would require $n(n-1) / 2$ maximum flow computations. Using a result in network flow theory we reduce the problem to one of computing $n$ maximum flow computations. 2) We are required to calculate the connectivity of the logical graph after each failure. In other words, connectivity computation has to be repeated on $m$ after-failure logical graphs. Normally this would require solving $m$ separate integer linear programs if we explicitly generate the logical graph after each one of the $m$ physical failures. See [6] where such an approach was taken in a different context. In our MILP we implicitly generate the after failure graphs so that just one MILP is enough to calculate the after-failure connectivity.

- Introduction of a new metric "logical capacity" and determining a routing that maximizes logical capacity: Given a survivable routing. Let us associate with each logical link $(s, t)$ a capacity equal to the capacity of the corresponding lightpath. Using these link capacities let us find the maximum $s-t$ flow between the nodes $s$ and $t$ of each logical link. The capacity of the logical graph is the minimum of the maximum $s-t$ flows over all pairs of nodes in the logical graph constructed. When a physical link fails, several logical links could fail. The 
larger the capacity of the logical graph under a given routing the more will be the capacity available for routing after a physical link failure. Thus the logical capacity is a measure of the available capacity after a failure. In Section V-B we provide an MILP that achieves a routing that maximizes the capacity of the logical graph. This is in contrast to our DRCN paper [3] that achieves a routing that maximizes the total capacity (sum of all logical link capacities, not logical capacity as defined above) available before and after a physical link failure.

- Determining a routing that maximizes MCLC (Minimum Cross Layer Cut): In [7] Lee and Modiano defined the concept of MCLC (minimum cross layer cut). MCLC value of a given survivable logical topology routing is the minimum number of physical link failures that would cause the logical layer to become disconnected. For example, if the MCLC value of a routing is 5, this means that the logical graph will remain connected after any set of 5 simultaneous physical link failures. In Section V-C we give an MILP that determines a routing that maximizes MCLC, in contrast to the work in [7] that determines the MCLC value of a given routing.

In Section VI we provide heuristics for all the optimization problems considered in earlier sections. In Section VII we provide extensive simulation results evaluating the performance of the heuristics and the corresponding MILPs.

\section{RELATED WORK}

A typical network reliability problem is to calculate efficiently the probability that a specified set of nodes can communicate with each other at a given time. The research works in the literature developed exact solution, Monte Carlo simulation, and the polynomial expression for network reliability estimations in single layer networks. [8] provided a survey for early exact factoring algorithms with domination theory. [9][10][11] utilized network transformation and reduction for network reliability estimation. Dikbiyik et al. [12] utilized preprovisioning/reprovisioning and hold-lightpath schemes which balanced optical network availability, resource efficiency, and protection through excess capacity management. These research works were for single-layer networks and cannot estimate cross-layer network reliability, as they assumed that link failures are independent. In fact, a single physical link failure may cause multiple failures in the logical network. Monte Carlo simulation was also used for estimating single-layer reliability for some fixed link failure probability. In [13], a Monte Carlo method is given to estimate the failure probability with random network link states. With Monte-Carlo simulation, network reliability could be approximated to an arbitrary accuracy. The computational performance of simulations was restricted by a large number of iterations and repeating them with different reliability indices. A randomized fully polynomial approximation scheme for the all-pairs reliability problem was given in [14]. A comprehensive study of combinatorial aspects of the network reliability problem may be found in [15]. Recently, there has been interest in the study of the network reliability problem when links and nodes are broken in a probabilistic manner. [16][17] discussed this problem and have several references to early related works.

As noted earlier, the survivability of a logical topology mapping can be guaranteed if the lightpaths in the physical topology corresponding to this mapping are all link-disjoint. Since finding mutually disjoint paths between multiple pairs of nodes is NP-complete [18], the cross-layer network reliability problem with survivability as an evaluation metric is also NPcomplete. Modiano and Narula-Tam [19] have given a necessary and sufficient condition for a cross-layer mapping to be survivable under a single physical link failure in IP-over-WDM networks, and formulated the problem as an Integer Linear Program (ILP). Todimala and Ramamurthy [20] adapted the concept of Shared Risk Link Group (SRLG) introduced in [21] and also computed the routing through an ILP formulation. Extensions of [19] were given by Kan et al. in [6], which discussed the relationship between survivable lightpath routing and spare capacity requirements on the logical links satisfying the original traffic demands after failures. Lin et al. [1][3] discussed rerouting disconnected logical links due to a physical link failure to maximize the sum of satisfied demands. [3] also studied spare capacity assignment to physical links to guarantee availability of capacities for rerouting after a failure. Similar to [3], Vadrevu et al. [22] also proposed ILPs and heuristics addressing survivability and reroutability for logical links with backup capacity sharing after any single physical link failure.

A common drawback of ILP approaches is that they are not scalable as the network size increases. Hence, heuristic approaches were designed to overcome such issues. Kurant and Thiran [23] proposed the Survivable Mapping by Ring Trimming (SMART) framework, which maps the links of certain subgraphs of the given logical graph into link-disjoint paths. Lee et al. [24] solved the same problem utilizing the concept of ear-decomposition in graph theory. Javed et al. [25][26] obtained improved heuristics based on SMART. Using duality theory in graphs, a generalized theory of logical topology survivability was given in [27][28][29]. A new structural approach based on logical protecting spanning tree set concept was introduced in [30]. Lin et al. [31] also presented an integrated approach to design survivable logical topology routing and localize physical link failures. Operations research techniques, such as column generation, have been incorporated into MILP formulations to solve similar problems. Jaumard and Hoang [32] proposed an MILP formulation and a solution approach based on the column generation technique which can generate exact solutions for different scales of logical networks. Two works related to disaster aware survivability in the context of cloud computing and survivable infrastructure designs are reported in [33][34].

\section{Weakly Survivable Routing Maximizing Total DEMAND SATISFIED: REVIEW OF AN MILP [1][3]}

We use the terms network and topology, edge and link, node and vertex, interchangeably throughout the paper. Let $G_{L}=\left(V_{L}, E_{L}\right)$ be a logical network and $G_{P}=\left(V_{P}, E_{P}\right)$ be a physical network in a cross-layer network. Let $(i, j)$ be a 
TABLE I

VARIABLES USED IN MILP FORMULATION WSR-MD

\begin{tabular}{|l|l|}
\hline Variable & Description \\
\hline$y_{i j}^{s t}$ & $\begin{array}{l}\text { binary variable indicates whether the logical link }(s, t) \in \\
E_{L} \text { is routed through the physical link }(i, j) \in E_{P} . \text { If } \\
\text { yes, } y_{i j}^{s t}=1, \text { otherwise, } y_{i j}^{s t}=0 .\end{array}$ \\
\hline$f_{i j}^{s t}$ & flow on physical link $(i, j)$ due to lightpath $(s, t)$ \\
\hline$r_{s t}^{i j}$ & $\begin{array}{l}\text { fractional variable for connectivity constraints. } r_{s t}^{i j}=0 \text { if } \\
\text { logical link }(s, t) \text { is routed through physical link }(i, j) .\end{array}$ \\
\hline$\rho_{s t}$ & $\begin{array}{l}\text { the capacity for the logical link }(s, t), \text { where } \rho_{s t} \text { is the } \\
\text { smallest capacity of links in the lightpath. }\end{array}$ \\
\hline$c_{i j}$ & capacity on the physical link $(i, j) \cdot c_{i j}=c_{j i}$. \\
\hline$d_{s t}$ & demand for the logical link $(s, t) \cdot d_{s t}=d_{t s}$. \\
\hline$M$ & a large positive number \\
\hline
\end{tabular}

physical link and $(s, t)$ be a logical link. Capacity on physical link $(i, j)$ is $c_{i j}$ and demand on logical link $(s, t)$ is $d_{s t}$. We assume that both the logical and physical networks are at least two-edge connected.

Definition 1: A logical topology mapping in a cross-layer network with logical topology $G_{L}=\left(V_{L}, E_{L}\right)$ and physical topology $G_{P}=\left(V_{P}, E_{P}\right)$ is weakly survivable if $G_{L}$ remains connected, after a link failure in $G_{P}$

In this section we review an MILP given in [3] to determine a weakly survivable routing in a cross-layer network that maximizes the sum of all logical demands satisfied subject to the limits on the capacities of physical link. This MILP formulation to be called WSR-MD is the basis of all the MILP formulations given in the rest of the paper.

WSR-MD: MILP to determine a weakly survivable logical topology routing maximizing the total demand.

$$
\begin{aligned}
& \max \sum_{(s, t) \in E_{L}} \rho_{s t} \\
& \text { s.t. } \sum_{(i, j) \in E_{P}} y_{i j}^{s t}-\sum_{(j, i) \in E_{P}} y_{j i}^{s t}=\left\{\begin{array}{cc}
1, & \text { if } i=s, \\
-1, & \text { if } i=t, \\
0, & \text { if } i \neq\{s, t\}
\end{array}\right. \\
& i \in V_{P},(s, t) \in E_{L} \\
& y_{i j}^{s t}+y_{j i}^{s t} \leq 1, \quad(i, j) \in E_{P},(s, t) \in E_{L} \\
& \sum_{(i, j),(j, i) \in E_{P}}\left(y_{i j}^{s t}+y_{j i}^{s t}\right) \leq 2,(s, t) \in E_{L} \\
& \sum_{(i, j) \in E_{P}} f_{i j}^{s t}-\sum_{(j, i) \in E_{P}} f_{j i}^{s t}=\left\{\begin{array}{cc}
\rho_{s t}, & \text { if } i=s, \\
-\rho_{s t}, & \text { if } i=t, \\
0, & \text { if } i \neq\{s, t\},
\end{array}\right. \\
& i \in V_{P},(s, t) \in E_{L} \\
& \rho_{s t} \leq d_{s t}, \quad(s, t) \in E_{L} \\
& f_{i j}^{s t} \leq M y_{i j}^{s t} \text { and } f_{j i}^{s t} \leq M y_{j i}^{s t} \text {, } \\
& (i, j) \in E_{P},(s, t) \in E_{L} \\
& \sum_{(s, t) \in E_{L}}\left(f_{i j}^{s t}+f_{j i}^{s t}\right) \leq c_{i j}, \quad(i, j) \in E_{P} \\
& \sum_{(s, t) \in E_{L}} r_{s t}^{i j}-\sum_{(t, s) \in E_{L}} r_{t s}^{i j}=\left\{\begin{array}{cl}
-1, & \text { if } s=v_{1}, \\
\frac{1}{\left|V_{L}\right|-1}, & \text { if } s \neq v_{1},
\end{array}\right. \\
& v_{1} \in V_{L},(i, j) \in E_{P} \\
& 0 \leq r_{s t}^{i j} \leq 1-\left(y_{i j}^{s t}+y_{j i}^{s t}\right) \text {, } \\
& (i, j) \in E_{P},(s, t) \in E_{L} \\
& 0 \leq r_{t s}^{i j} \leq 1-\left(y_{i j}^{s t}+y_{j i}^{s t}\right) \text {, } \\
& (i, j) \in E_{P},(s, t) \in E_{L} \\
& y_{i j}^{s t} \in\{0,1\}, r_{i j}^{s t}, f_{i j}^{s t}, \rho_{s t} \geq 0 \text {, } \\
& (s, t) \in E_{L},(i, j) \in E_{P}
\end{aligned}
$$

Constraint (2) with binary variable $y_{i j}^{s t}$ provides a cross-layer routing for each logical link $(s, t)$ with single unit flow. Constraint (3) guarantees that a logical-layer routing is not routed through a physical-layer link in its two directions. Constraint (4) eliminates other loops by avoiding a logical layer routing to revisit the same node on the physical routing. Without constraints (3) and (4), constraint (2) itself will not be able to avoid self-loops and re-entry of the same node. Constraints (5)-(6) push a flow of value $\rho_{s t}$ through the logical layer routing selected for logical link $(s, t)$. Constraint (7) guarantees that each physical link $(i, j)$ carries flow only if the logical layer routing is routed through at least one direction of the physical link. Here $M$ is a very large number greater than the maximum link capacity. Constraint (8) requires that the total flow of all the logical layer routings carried by each physical layer link is no more than the corresponding physical link capacity. Constraints (9)-(11) ensure that the logical network after the failure of each physical link contains a spanning tree. Constraint (9) is used to guarantee the existence of a logical spanning structure after a physical link failure. Constraints (10) and (11) make sure that the spanning tree 
structure is composed of the logical edges not disconnected by the failure of a physical link $(i, j)$. We wish to note that the survivability constraints (9)-(11) were first employed in Deng et al.'s paper [35].

\section{Minimum Logical Topology Augmentation for Guaranteed Weakly Survivable Routing}

Given a cross-layer network, it is possible that the logical topology does not permit a weakly survivable routing. In such a case we need to add additional logical links to guarantee the existence of a weakly survivable routing. This augmentation problem was earlier considered in [4][5]. In this section we now show how to enhance the WSR-MD algorithm to accommodate steps to augment the logical network with minimum number of additional links so that the augmented network admits a weakly survivable routing. It is shown in [4] that such an augmentation is always possible if the physical network is at least 3-edge connected.

Let $L_{L}$ be the collection of all ordered pairs of vertices in the logical network. Some of these ordered pairs may not represent links in the original logical network. We introduce auxiliary variable $h_{s t}$ to indicate whether $(s, t)$ is a link in the logical network. $(s, t)$ could be a link in the original logical network or was added during augmentation. We let $g_{s t}$ be the variable which indicates whether $(s, t)$ is a link in the original logical network $G_{L}$. Then, we have

\section{Logical link augmentation constraints:}

$$
\begin{aligned}
& g_{s t} \leq h_{s t}, \quad(s, t) \in L_{L} \\
& y_{i j}^{s t} \leq h_{s t}, \quad(s, t) \in L_{L},(i, j) \in E_{P} \\
& \sum_{(i, j) \in E_{P}} y_{i j}^{s t}-\sum_{(j, i) \in E_{P}} y_{j i}^{s t}=\left\{\begin{array}{cc}
h_{s t}, & \text { if } i=s, \\
-h_{s t}, & \text { if } i=t, \\
0, & \text { if } i \neq\{s, t\},
\end{array}\right. \\
& i \in V_{P},(s, t) \in L_{L} \\
& g_{s t}, h_{s t} \in\{0,1\},(s, t) \in L_{L} .
\end{aligned}
$$

Constraint (12) builds the connection between the indicator $g_{s t}$ and variable $h_{s t}$. If $(s, t)$ is a link in the given logical network (that is, $g_{s t}=1$ ), then, $(s, t)$ exists in the augmented network (that is, $h_{s t}=1$ ). So, in this case, $g_{s t} \leq h_{s t}$. If $g_{s t}=0,(12)$ is trivially satisfied. Constraint (13) indicates that a logical routing is generated only if $(s, t)$ is in the augmented network. Constraint (14), similar to constraint (2), provides logical routings for original and augmented links in the logical topology.

The MILP formulation for minimum logical topology augmentation problem is as follows.

WSR-MLA: Minimal logical topology augmentation for guaranteed weakly survivable routing

$$
\min \sum_{(s, t) \in L_{L}} h_{s t}
$$

s.t. Constraints (3),(4),(9) to (11), (12)-(15)

\section{Weakly Survivable Routing under Cross-LayeR METRICS}

In this section we develop MILP formulations to determine a survivable logical topology routing that maximizes one of three cross layer metrics.

\section{A. Maximizing the After-failure Connectivity of the Logical} Topology

The connectivity of a graph is the smallest number of edges whose removal disconnects the graph [36]. Our interest is to find a survivable logical topology routing that maximizes the after-failure connectivity of the logical topology after a single physical link failure. This is equivalent to finding a survivable logical routing that maximizes the number of edges remaining in any cut of $G_{L}$ after any physical link failure.

In the following $G_{L}^{i j}$ will refer to the graph that results from deleting those links in $G_{L}$ which get broken when physical link $(i, j)$ fails. Let $k_{i j}(s, t)$ denote the maximum number of link-disjoint paths between logical nodes $s$ and $t$ in $G_{L}^{i j}$ which is the $s-t$ connectivity of $G_{L}^{i j}$ [36]. Then the after-failure connectivity of $G_{L}$ under a given routing is $\min _{s, t \in V_{L},(i, j) \in E_{P}}\left\{k_{i j}(s, t)\right\}$. Our objective is to determine a survivable logical routing that maximizes the after-failure connectivity of $G_{L}$.

Let $k_{v w}^{i j}(s, t)$ denote the flow on link $(v, w) \in E_{L}$ due to an $s-t$ flow in $G_{L}^{i j}$. Picking a node $s_{0}$, the following MILP will calculate the maximum number of link disjoint $s_{0}-t$ paths for all $t \in V_{L}$ in each $G_{L}^{i j}$, and then pick minimum of these numbers as the after-failure connectivity of $G_{L}$. Note that we do not have to calculate the maximum number of link disjoint paths between all pairs of logical nodes $s$ and $t$. See [37] for correctness of this approach.

$$
\begin{aligned}
& \text { Given } s_{0} \in V_{L}, t \in V_{L} \\
& \max k \\
& \sum_{(v, w) \in E_{L}} k_{v w}^{i j}\left(s_{0}, t\right)-\sum_{(w, v) \in E_{L}} k_{w v}^{i j}\left(s_{0}, t\right) \\
& =\left\{\begin{array}{cc}
k_{i j}\left(s_{0}, t\right), \quad \text { if } v=s_{0}, \\
-k_{i j}\left(s_{0}, t\right), \quad \text { if } v=t, \\
0, \quad \text { if } v \neq\left\{s_{0}, t\right\}, & v \in V_{L} \\
(i, j) \in E_{P} & v \in V_{L}
\end{array}\right. \\
& k_{v w}^{i j}\left(s_{0}, t\right)+k_{w v}^{i j}\left(s_{0}, t\right) \leq 1, \\
& \quad(v, w),(w, v) \in E_{L},(i, j) \in E_{P}, t \in V_{L} \\
& \left(\sum_{v w}^{i j}\left(s_{0}, t\right)+k_{w v}^{i j}\left(s_{0}, t\right)\right) \leq 2,(i, j) \in E_{P} \\
& k_{v w}^{i j}\left(s_{0}, t\right) \leq 1-y_{i j}^{v w}, \quad(i, j) \in E_{P},(v, w) \in E_{L} \\
& k \leq k_{i j}\left(s_{0}, t\right), \quad(i, j) \in E_{P} \\
& k_{v w}^{i j} \in \mathcal{B}, k_{i j}\left(s_{0}, t\right) \in \mathcal{Z}^{+}, \quad(i, j) \in E_{P},(v, w) \in E_{L}
\end{aligned}
$$

Note that constraints (18)-(19) guarantee that logical routings of $\left(s_{0}, t\right)$ generated by constraint (17) are link-disjoint. Constraint (20) guarantees only edges that are not broken due to $(i, j) \in E_{P}$ failure are considered when calculating $k_{i j}\left(s_{0}, t\right)$. This interesting feature helps us avoid to calculate $G_{L}^{i j}$ explicitly for each $(i, j)$, thereby, allowing us to provide one single formulation instead of several formulations, one for each physical link $(i, j)$.

Now using (16) in place of (1) and adding the constraints (17)-(22) to the MILP (WSR-MD) we get the following 
MILP WSR-AFC that determines a survivable logical topology routing that maximizes the after-failure connectivity of $G_{L}$.

WSR-AFC: Weakly survivable routing maximizing after failure connectivity of logical topology

$$
\begin{aligned}
& \max k \\
& \text { s.t. Constraints (2) - (4), (9) - (11), (17) - (22) }
\end{aligned}
$$

We wish to note that after-failure connectivity is a measure of the ability of a logical topology routing to provide survivability against multiple failures. If this connectivity is larger, then one can expect the routing to provide survivability against a larger number of physical link failures.

\section{B. Maximizing the Capacity of the Logical Topology}

Given a routing that achieves a demand of $\rho_{v w}$ on logical link $(v, w)$. Let $\Psi(s, t)$ be the maximum flow between any pair of nodes $s, t \in V_{L}$ while treating $\rho_{v w}$ as the capacity of link $(v, w) \in E_{L}$. Then we define the capacity of $G_{L}$ under the given routing as $\min _{s, t \in V_{L}}\{\Psi(s, t)\}$. Our interest is to determine a survivable logical routing that maximize logical capacity. We proceed as follows.

Let $\bar{f}_{v w}(s, t)$ be the flow on logical link $(v, w)$ due to a flow $\Psi(s, t)$ between nodes $s$ and $t$ in $V_{L}$. The following linear program determines $\Psi(s, t)$ for each pair of nodes $s$ and $t$ in $V_{L}$ and picks the minimum of these maximum flows which is the capacity of $G_{L}$.

$$
\begin{aligned}
& \sum_{(v, w) \in E_{L}} \bar{f}_{v w}(s, t)-\sum_{(w, v) \in E_{L}} \bar{f}_{w v}(s, t) \\
& =\left\{\begin{array}{ccc}
\Psi(s, t), & \text { if } v=s, & s, t, v \in V_{L} \\
-\Psi(s, t), & \text { if } v=t, & s, t, v \in V_{L} \\
0, & \text { if } v \neq\{s, t\}, & s, t, v \in V_{L}
\end{array}\right. \\
& \bar{f}_{v w}(s, t)+\bar{f}_{w v}(s, t) \leq \rho_{v w}, \quad s, t \in V_{L},(v, w) \in E_{L} \\
& \tilde{f} \leq \Psi(s, t), \\
& \bar{f}_{v w}(s, t), \Psi(s, t), \tilde{f} \geq 0, \quad s, t \in V_{L}
\end{aligned}
$$

Using the objective (23) in place of (1) and adding the constraints (24)-(27) to the MILP (WSR-MD), we get the following MILP that determines a survivable logical topology routing that maximizes the capacity of the logical topology $G_{L}$.

WSR-MLC:Weakly survivable routing with maximal logical capacity

$$
\begin{aligned}
& \max \tilde{f} \\
& \text { s.t. Constraints (2) to (11), and (24) to (27) }
\end{aligned}
$$

\section{Maximizing the Min-Cross-Layer Cut}

Given a logical topology routing $\mathcal{R}$, the minimum crosslayer cut $M C L C(\mathcal{R})$ of $\mathcal{R}$ is defined in [7] as the smallest number of physical link failures that will disconnect the logical topology. We wish to find a routing $\mathcal{R}$ that has the maximum value for $M C L C(\mathcal{R})$. If this maximum value is greater than or equal to one, then that routing will be survivable.
In [7] Lee and Modiano showed that finding a routing that has the largest MCLC value is NP-complete. So they presented an ILP to find a routing that minimizes a quantity whose reciprocal gives a lower bound on the MCLC value. Lee and Modiano [7] also presented an ILP $M_{M C L C}$ to find the MCLC value of a given logical topology routing. We now show how $M_{M C L C}$ can be integrated with the constraints (1)(5) of Section III to yield an ILP for finding a logical topology routing with the largest MCLC value. This ILP is given in (28) - (35).

\section{WSR-MCLC: Weakly survivable routing with maximum MCLC value}

$$
\begin{aligned}
& \min \lambda \\
& \text { s.t. }(2)-(4) \\
& \lambda \geq \sum_{(i, j) \in E_{P}} \nu_{i j} \\
& \xi_{i j}^{s t} \leq \nu_{i j}, \quad(i, j) \in E_{P} \\
& \xi_{i j}^{s t} \leq y_{i j}^{s t}, \quad(s, t) \in E_{L},(i, j) \in E_{P} \\
& \xi_{i j}^{s t} \geq \nu_{i j}+y_{i j}^{s t}-1,(s, t) \in E_{L},(i, j) \in E_{P} \\
& \left|\psi_{t}-\psi_{s}\right| \leq \sum_{(i, j) \in E_{P}} \xi_{i j}^{s t},(s, t) \in E_{L} \\
& \sum_{s \in V_{L}} \psi_{s} \geq 1 \quad s, t \in V_{L},(s, t) \in E_{L},(i, j) \in E_{P} \\
& \psi_{0}=0 \quad
\end{aligned}
$$

First we note that the variables $\nu_{i j}, y_{i j}^{s t}$, and $\psi_{t}$ used in our ILP correspond to the variables $y_{i j}, f_{i j}^{s t}$, and $d_{t}$ used in $M_{M C L C}$ of [7]. Second, we point out constraint (3) in [7].

$$
\psi_{t}-\psi_{s} \leq \sum_{(i, j) \in E_{P}} \nu_{i j} y_{i j}^{s t}, \quad \forall(s, t) \in E_{L}
$$

should be replaced with.

$$
\left|\psi_{t}-\psi_{s}\right| \leq \sum_{(i, j) \in E_{P}} \nu_{i j} y_{i j}^{s t}, \quad \forall(s, t) \in E_{L}
$$

due to the fact that the identification whether $s$ and $t$ is connected, is evaluated by whether $s$ is disconnected from $t$ or $t$ is disconnected from $s$. To obtain these bi-direction information, absolute value is used as shown in constraint (36).

The main difficulty in using $M_{M C L C}$ is that it has a constraint that involves the nonlinear term $\nu_{i j} y_{i j}^{s t}$. This constraint needs to be replaced by an equivalent set of constraints. For this purpose we introduced a new variable $\xi_{i j}^{s t}$ which indicates whether the routing of $(s, t)$ is impacted by the failure of physical link $(i, j)$. With this new variable constraints (29)(31) represent a set of constraints equivalent to the following constraint

$$
\sum_{(i, j) \in E_{P}} \xi_{i j}^{s t}=\sum_{(i, j) \in E_{P}} \nu_{i j} y_{i j}^{s t}, \quad(s, t) \in E_{L} .
$$

This follows because $y_{i j}^{s t}=1 \Rightarrow \nu_{i j} y_{i j}^{s t}=\nu_{i j}$ and $\xi_{i j}^{s t}=\nu_{i j}$ because of (29) and (31). $y_{i j}^{s t}=0 \Rightarrow \nu_{i j} y_{i j}^{s t}=0$ and $\xi_{i j}^{s t}=0$ because of (30). 
Then constraint (32) can be further linearized as

$$
\begin{aligned}
& \psi_{t}-\psi_{s} \leq \sum_{(i, j) \in E_{P}} \xi_{i j}^{s t},(s, t) \in E_{L} \\
& \psi_{s}-\psi_{t} \leq \sum_{(i, j) \in E_{P}} \xi_{i j}^{s t},(s, t) \in E_{L} .
\end{aligned}
$$

\section{HEURISTICS}

The MILP formulations presented in Sections IV and $\mathrm{V}$ require high execution times, though they produce optimal/feasible solutions. To mitigate the computational complexity of these formulations, we present in this section heuristics for all the problems considered.

\section{A. Heuristic for logical topology augmentation}

The heuristic augments the given logical topology with additional links so that a survivable routing is guaranteed for the augmented logical topology. The routing corresponding to each logical link is also generated. This procedure is provided in Algorithm 1.

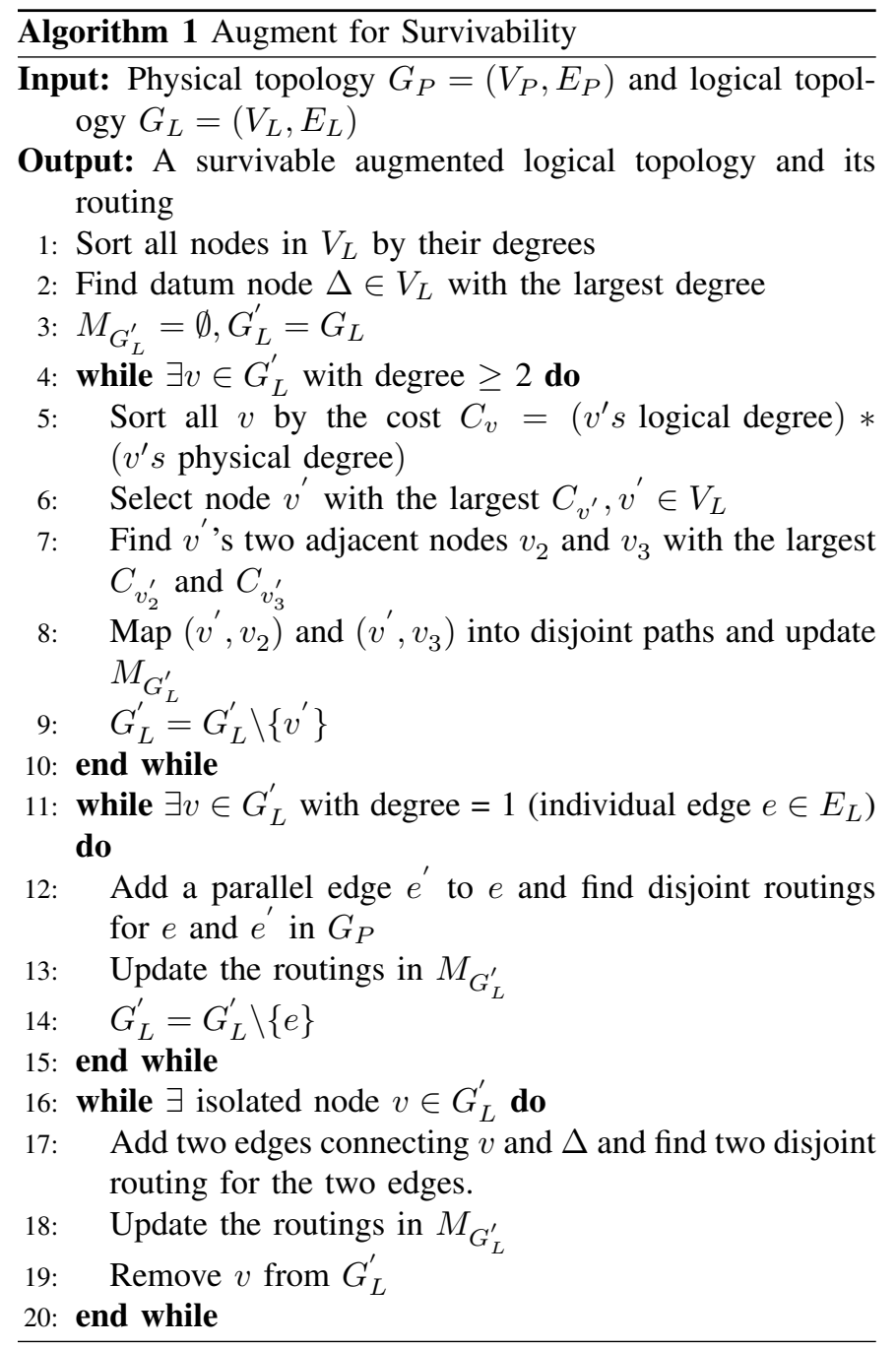

The heuristic first sorts all logical nodes by their degrees, and a datum node with the maximum degree is assigned and denoted as $\Delta$. While there exists logical node $v$ with degree $\geq 2$, the heuristic assigns each logical node $v$ with degree $\geq 2$ a cost $C_{v}=$ (degree of logical node) * (degree of corresponding physical node). The heuristic then selects the logical node with the largest $C_{v}$ and chooses two of $v$ 's adjacent nodes $v_{1}$ and $v_{2}$ with the largest $C_{v_{1}}$ and $C_{v_{2}} .\left(v, v_{1}\right)$ and $\left(v, v_{2}\right)$ are then mapped into disjoint paths in the physical topology. After that, $v$ is removed from the logical topology. This procedure is repeated till no logical nodes with degree $\geq 2$ are left. Next, the heuristic picks a node $v$ from the remaining logical topology with degree $=1$, i.e., an edge $(u, v)$. An edge $\left(u, v^{\prime}\right)$ parallel to $(u, v)$ is then added and disjoint mappings for $(u, v)$ and $\left(u, v^{\prime}\right)$ in the physical topology are found. This procedure is executed until the elimination of all logical nodes with degree $=1$. After the previous steps, if there exists nodes $v$ with degree $=0$, two parallel edges connecting $v$ and $\Delta$ are added and they are mapped disjointly in the physical topology. The heuristic is provided in Algorithm 1. Proof of correctness of Algorithm 1 may be found in [4].

As regards computational complexity, the complexity of finding a pair of link-edge disjoint paths dominates the time required, which is $O\left(\mathrm{~m}^{3 / 2}\right)$. So, the overall complexity is $O\left(n m^{3 / 2}\right)$.

\section{B. Heuristics for maximizing logical capacity}

We present a heuristic for maximizing logical capacity in Algorithm 2. To guarantee survivability, the heuristic would still augment the logical topology with additional links. Steps 1-6 are the same as those in Algorithm 1. After selecting the candidate node $C_{v}$ with degree $\geq 2$ and the largest $C_{v}$, instead of mapping its adjacent nodes $v_{1}$ and $v_{2}$ with the largest $C_{v_{1}}$ and $C_{v_{2}}$, the $C_{v_{i}}$ 's are included in a priority list. The heuristic selects two nodes at a time with the highest $C_{v_{i}}$ (in descending order), finds their disjoint mappings, and determines the minimal capacity of the routing. Among all the routings generated, the one that maximizes the minimal capacity is selected. This procedure is repeated till no logical node with degree $\geq 2$ is left.

After a survivable routing is generated, to determine the maximal logical capacity, we first push the flow for all logical demands. This step is done by repeatedly pushing unit flow for each logical demand until the physical capacity cannot carry more logical demand. Here the physical edge capacity of an edge would be updated if the unit flow is routed through this edge. After the above step, the maximum flow is pushed for every logical node pair.

The complexity is dominated by step 9 in in Algorithm 2. At most $n^{2}$ pairs of adjacent nodes are picked, and for each pair edge-disjoint paths are to be found. This requires $O\left(n^{2} m^{3 / 2}\right)$ time. Since this is done for each node, the overall complexity is $O\left(n^{3} m^{3 / 2}\right)$.

The complexity of Algorithms 1 and 2 are highly conservative as can be seen from the simulation results.

\section{Heuristics for Maximizing the After-Failure Connectivity and the MCLC}

These heuristics in Algorithms 3 and 4 start with a spanning tree $t$, and map their links into paths using a shortest path 


$\overline{\text { Algorithm } 2 \text { Survivability and Maximizing Logical Topology }}$
Capacity

Input: Physical topology $G_{P}=\left(V_{P}, L_{P}\right)$, logical topology $G_{L}=\left(V_{L}, E_{L}\right)$

Output: A logical routing with the maximum capacity

1: Sort all nodes in $V_{L}$ by their degrees

2: Find datum node $\Delta \in V_{L}$ with the largest degree

3: $M_{G_{L}^{\prime}}=\emptyset, G_{L}^{\prime}=G_{L}$

4: while $\exists v \in G_{L}^{\prime}$ with degree $\geq 2$ do

5: Sort all $v$ by the cost $C_{v}=\left(v^{\prime} s\right.$ logical degree $) *$ $\left(v^{\prime} s\right.$ physical degree)

6: Select node $v^{\prime}$ with the largest $C_{v^{\prime}}, v^{\prime} \in V_{L}$

7: Sort $v^{\prime}$ adjacent node $v_{i}^{\prime}$ by $C_{v_{i}^{\prime}}$

8: for Each $v^{\prime}$ 's adjacent node pair $v_{1}^{\prime}, v_{2}^{\prime}$ from sorted $C_{v_{i}^{\prime}}$ list (in descending order) do

9: $\quad \operatorname{Map}\left(v^{\prime}, v_{1}^{\prime}\right),\left(v^{\prime}, v_{2}^{\prime}\right)$ disjointly and decide the minimum capacity physical link on the lightpath

10: Record the sum of minimum capacities of the two lightpaths

11: end for

12: Select the node pairs $v_{1}^{\prime}, v_{2}^{\prime}$ with the largest sum of minimum capacities and update $M_{G_{L}^{\prime}}$ with lightpaths of $\left(v^{\prime}, v_{1}^{\prime}\right),\left(v^{\prime}, v_{2}^{\prime}\right)$

13: $G_{L}^{\prime}=G_{L}^{\prime} \backslash\left\{v^{\prime}\right\}$

14: end while

15: while $\exists v \in G_{L}^{\prime}$ with degree $=1$ (individual edge $e \in E_{L}$ ) do

16: Add a parallel edge $e^{\prime}$ to $e$ and find disjoint routings for $e$ and $e^{\prime}$ in $G_{P}$

17: Update the routings in $M_{G_{L}^{\prime}}$

18: $G_{L}^{\prime}=G_{L}^{\prime} \backslash\{e\}$

19: end while

20: while $\exists$ isolated node $v \in G_{L}^{\prime}$ do

21: Add two edges connecting $v$ and $\Delta$ and find two disjoint routing for the two edges.

22: Update the routings in $M_{G_{L}^{\prime}}$

23: $\quad$ Remove $v$ from $G_{L}^{\prime}$

24: end while

25: while $\exists$ unsatisfied logical demand of $u \in E_{L}$ and residual physical capacity supporting the demand do

26: Push unit demand between edge nodes of $u$ through $u$ 's routing

27: Update physical link capacity

28: end while

29: for all $v, w \in V_{L}, v \neq w$ do

30: Push the maximum flow between $(v, w)$

31: end for

32: Return the maximum flow among all $v, w \in V_{L}$

algorithm At the end of this step all the physical edges that were not used in this mapping of the links in the tree are stored in a set $C B(t)$.

Here a block refers to the set of co-tree edges with respect to a spanning tree edges. We have to make efforts that locks are not disjoint. We want them to overlap as much as possible so that an edge appears in more than one block. If this could be done then after a physical link failure several trees will remain connected increasing the connectivity of the logical network after a failure. $\mathcal{W}$ is a large number.

Algorithm (3): Maximizing After-Failure Connectivity

After finding mappings of all links in a tree $t_{i}$ the set $C B\left(t_{i}\right)$ is created. All the edges in $C B\left(t_{i}\right)$ are assigned a large $\mathcal{W}$ to discourage their selection for the mappings in the subsequent tree. This is to ensure that $C B$ blocks for two subsequent trees overlap as much as possible, This will guarantee that after the failure of an edge that is in two consecutive $C B$ 's at least two trees will remain resulting in increased after-failure connectivity.

Algorithm (4): Maximizing MCLC Value

In this heuristic all the edges that are used in the mapping of a logical link in a tree $t_{i}$ are assigned a large weight so that these edges are discouraged from entering the $C B$ for $t_{i}$. This is to create as large as a $C B\left(t_{i}\right)$ as possible. A larger $C B$ means that multiple failures of the edge in $C B$ will not disconnect the tree $t_{i}$ thereby increasing the MCLC value.

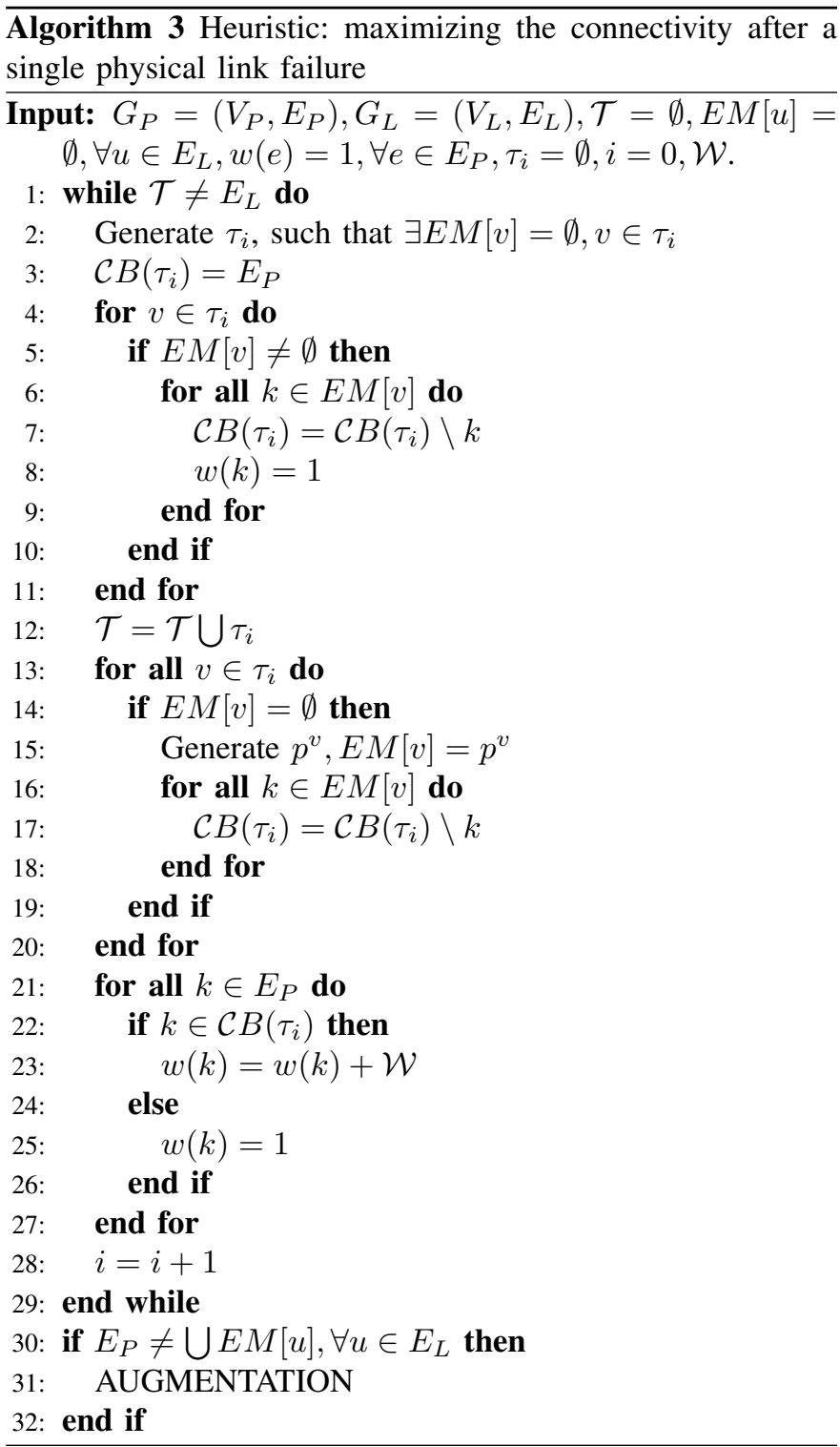




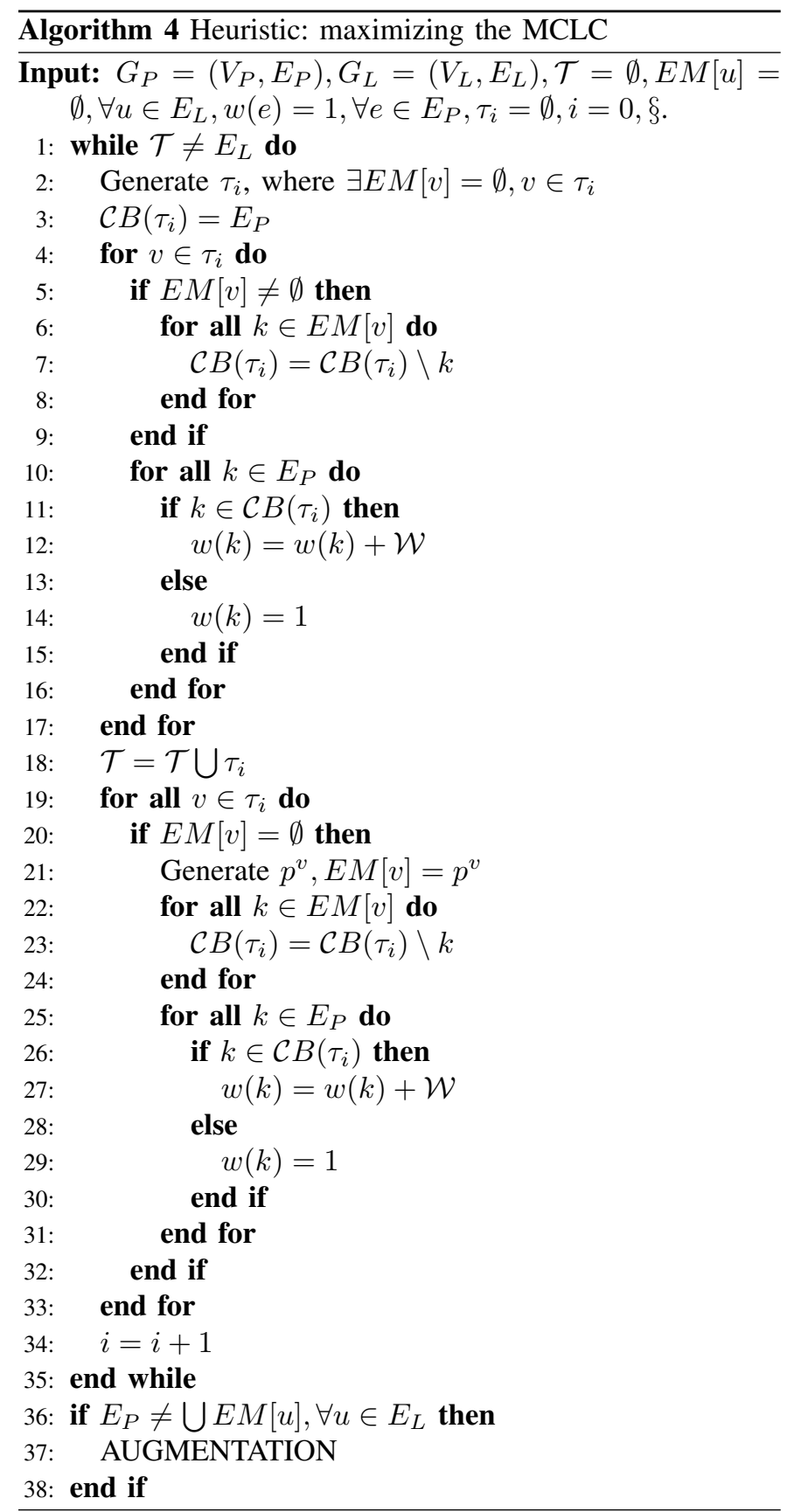

\section{Computational Results}

In this section, we report our computational results evaluating the effectiveness and performance of our exact solution approaches (MILP formulations) and heuristics for (1) minimizing logical topology augmentation for weak survivability; (2) survivable routing for maximizing the logical topology capacity; (3) survivable routing for maximizing the connectivity after a single physical link failure; and (4) maximizing the minimal cross-layer cutset.

\section{A. Experimental Setup}

The testing cases for both exact solution approaches (MILP formulations) and heuristics algorithms are as follows. We adopted networks introduced in [38][39] as physical topologies. They are DFN, EURO1 (E1), EURO2 (E2), G3, G6, and NSF as shown in Figures 2-7. Two groups of logical topologies are constructed: (a) two-connected planar graph, and (b) the more sparse networks, delaunay triangulation, and spanning tree, denoted as "2CON", "DELA", and "TREE", respectively.

DELA and TREE are used to test the topology augmentation MILP and the heuristic. Two-connected planar graphs are used to test all metrics. Note here that links are randomly removed from 2-connected and delaunay triangulation logical topologies to generate the tested logical networks. For all constructed logical topologies, the logical topologies' nodes are subsets of physical nodes, that is, $\left|V_{L}\right|=\left\lfloor 0.5 *\left|V_{P}\right|\right\rfloor$ and $\left\lfloor 0.7 *\left|V_{P}\right|\right\rfloor$. We let $r_{L / P}$ denote the "logical to physical node ratio." We used $r_{L / P}=0.5$ or 0.7 . The detailed information for physical and logical topologies are listed in Tables II and III. In all tested cases, capacities and demands assigned to physical and logical links are randomly generated following uniform distributions in $[1,10]$ and $[1,100]$, respectively. We run 10 cases for each tested scenario and report the average performance of these 10 cases.

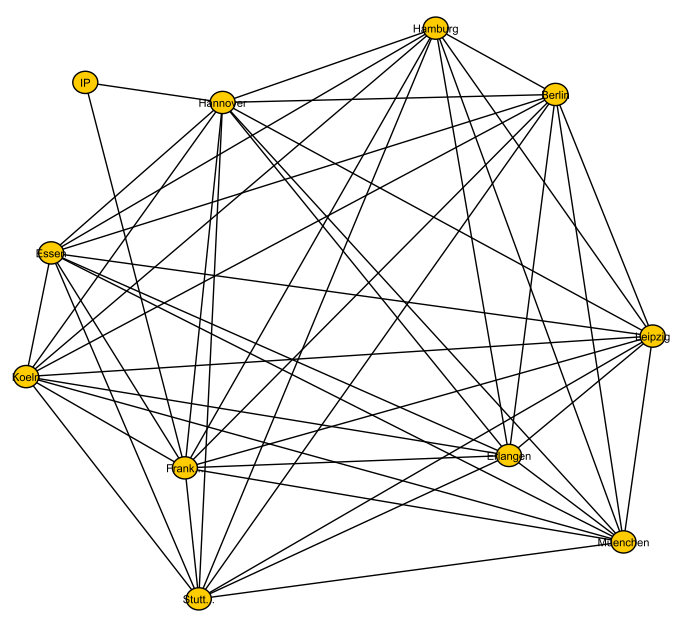

Fig. 2. DFN

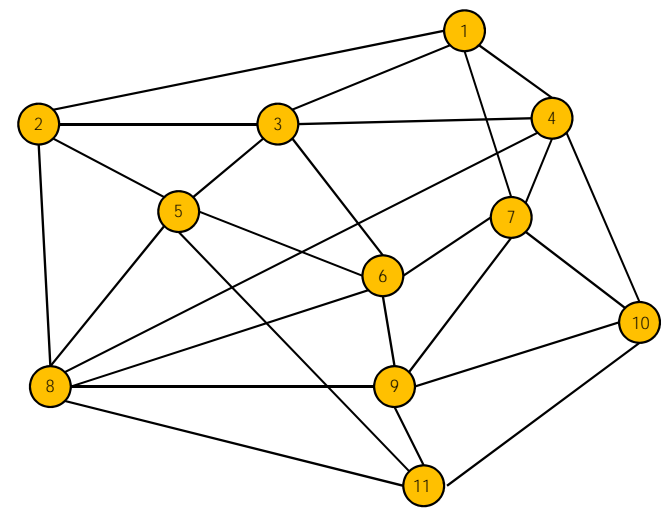

Fig. 3. EURO 1 


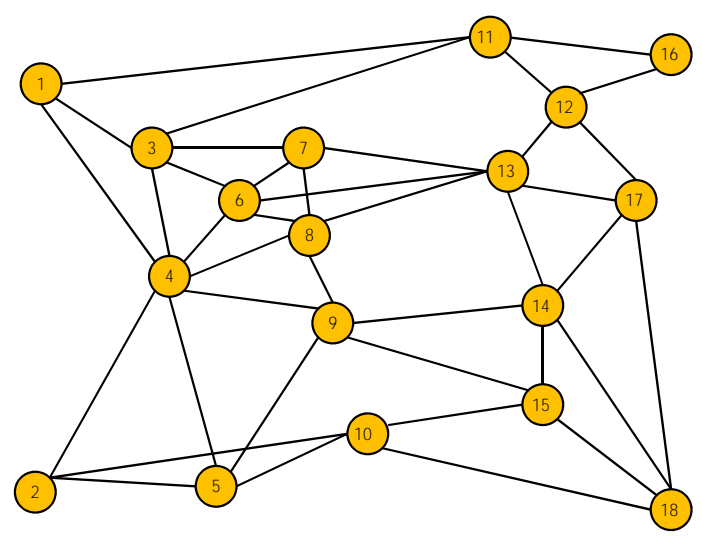

Fig. 4. EURO 2

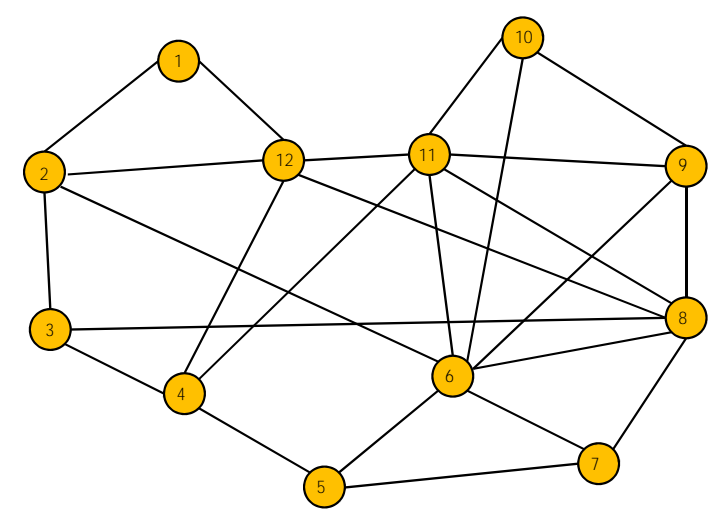

Fig. 5. G3

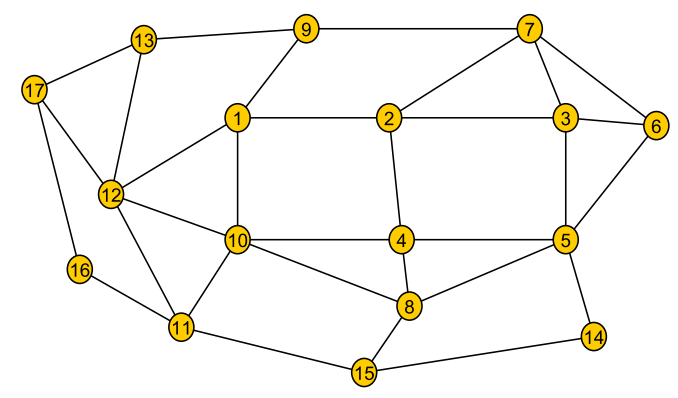

Fig. 6. G6

We used CPLEX 12.4 to run the MILP formulations on a machine with a quad-core(with hyper-threading) Intel Core i7$3770 \mathrm{~K}$ processor and $16 \mathrm{~GB}$ memory. We assigned four threads to solve each MILP problem and limited the maximum execution time to be 30 minutes. The heuristics were implemented using LEMON library [40], which ran in a single thread during execution.

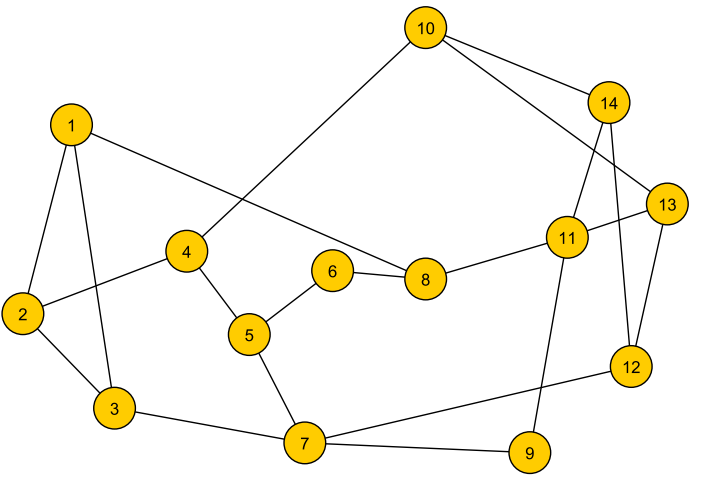

Fig. 7. NSF

TABLE II

PHYSICAL TOPOLOGIES INFORMATION

\begin{tabular}{l|c|c|c|c|c}
\hline PhyG & Nodes & Edges & MinDeg & MaxDeg & AvgDeg \\
\hline DFN & 11 & 47 & 2 & 10 & 8.55 \\
\hline E1 & 11 & 26 & 4 & 6 & 4.73 \\
\hline E2 & 18 & 39 & 2 & 7 & 4.33 \\
\hline G3 & 12 & 25 & 2 & 7 & 4.17 \\
\hline G6 & 17 & 31 & 2 & 5 & 3.65 \\
\hline NSF & 14 & 21 & 2 & 4 & 3 \\
\hline
\end{tabular}

\section{B. Experimental Results}

First, we present the results for the minimizing logical network augmentation. Note that logical network augmentation is triggered only when cross-layer survivable route cannot be generated. We consider "DELA" and "TREE" as logical networks, which are less dense than " $2 \mathrm{CON}$ ". The performance results for MILP formulation and heuristic for minimizing logical network augmentation for weak survivability are presented in Table IV. We let "PhyG ", "LogG", " $r_{L / P}$ ", "AugEdge" and "Time" represent the names of tested physical network, logical network, the ratio of the number of logical nodes over that of physical nodes, the number of augmented logical edges, and computational time (in seconds.) In all cases, the WSR-MLA formulation does not augment the logical network. The computational time of the heuristic is much less than the MILP approach. We note that for the same physical network, the denser the logical graph (as measured by the ratio $m / n^{2}$ ), the less the number of edges required for augmentation. This is because a denser graph can afford to tolerate more link failures before it becomes disconnected. This can be seen from the simulation results. In fact, DELA which is denser then TREE requires no augmentation at all.

Next, we present the performance of the MILPs and heuristics for all four cross-layer evaluation metrics with " $2 \mathrm{CON}$ " (with logical physical node ratio, 0.5 and 0.7,) as logical topologies. Computational results are reported in Table V and VI. For all testing cases, heuristic algorithms ran significantly faster than exact solution approaches (MILP formulations). For all evaluation metrics except augmentation, we report the average computational time of MILPs which do not need logical augmentation. As we can see, heuristics are much faster 
TABLE III

LOGICAL TOPOLOGIES INFORMATION

\begin{tabular}{|c|c|c|c|c|c|c|c|}
\hline PhyG & $\log G$ & $r_{L / P}$ & $V_{L}$ & $E_{L}$ & MinDeg & MaxDeg & AvgDeg \\
\hline \multirow[t]{6}{*}{ DFN } & \multirow[t]{2}{*}{$2 \mathrm{CON}$} & 0.5 & 6 & 7 & 2 & 4 & 2.33 \\
\hline & & 0.7 & 8 & 10 & 2 & 4 & 2.5 \\
\hline & \multirow{2}{*}{ DELA } & 0.5 & 6 & 47 & 5 & 31 & 15.67 \\
\hline & & 0.7 & 8 & 47 & 10 & 13 & 11.75 \\
\hline & \multirow{2}{*}{ TREE } & 0.5 & 6 & 5 & 1 & 2 & 1.67 \\
\hline & & 0.7 & 8 & 7 & 1 & 3 & 1.75 \\
\hline \multirow[t]{6}{*}{$\mathrm{E} 1$} & \multirow[t]{2}{*}{$2 \mathrm{CON}$} & 0.5 & 6 & 7 & 2 & 4 & 2.33 \\
\hline & & 0.7 & 8 & 10 & 2 & 6 & 2.5 \\
\hline & \multirow[t]{2}{*}{ DELA } & 0.5 & 6 & 26 & 5 & 12 & 8.67 \\
\hline & & 0.7 & 8 & 26 & 5 & 9 & 6.5 \\
\hline & \multirow[t]{2}{*}{ TREE } & 0.5 & 6 & 5 & 1 & 3 & 1.67 \\
\hline & & 0.7 & 8 & 7 & 1 & 3 & 1.75 \\
\hline \multirow[t]{6}{*}{$\mathrm{E} 2$} & \multirow[t]{2}{*}{$2 \mathrm{CON}$} & 0.5 & 9 & 11 & 2 & 4 & 2.44 \\
\hline & & 0.7 & 13 & 15 & 2 & 4 & 2.31 \\
\hline & \multirow{2}{*}{ DELA } & 0.5 & 9 & 39 & 7 & 11 & 8.67 \\
\hline & & 0.7 & 13 & 39 & 3 & 9 & 6 \\
\hline & \multirow[t]{2}{*}{ TREE } & 0.5 & 9 & 8 & 1 & 3 & 1.78 \\
\hline & & 0.7 & 13 & 12 & 1 & 3 & 1.85 \\
\hline \multirow[t]{6}{*}{ G3 } & \multirow[t]{2}{*}{$2 \mathrm{CON}$} & 0.5 & 6 & 7 & 2 & 4 & 2.33 \\
\hline & & 0.7 & 8 & 8 & 2 & 2 & 2 \\
\hline & \multirow[t]{2}{*}{ DELA } & 0.5 & 6 & 25 & 5 & 11 & 8.33 \\
\hline & & 0.7 & 8 & 25 & 5 & 8 & 6.25 \\
\hline & \multirow[t]{2}{*}{ TREE } & 0.5 & 6 & 5 & 1 & 2 & 1.67 \\
\hline & & 0.7 & 8 & 7 & 1 & 3 & 1.75 \\
\hline \multirow[t]{6}{*}{ G6 } & \multirow[t]{2}{*}{$2 \mathrm{CON}$} & 0.5 & 9 & 9 & 2 & 2 & 2 \\
\hline & & 0.7 & 12 & 14 & 2 & 4 & 2.33 \\
\hline & \multirow[t]{2}{*}{ DELA } & 0.5 & 9 & 31 & 3 & 10 & 6.89 \\
\hline & & 0.7 & 12 & 31 & 3 & 7 & 5.17 \\
\hline & \multirow[t]{2}{*}{ TREE } & 0.5 & 9 & 8 & 1 & 2 & 1.78 \\
\hline & & 0.7 & 12 & 11 & 1 & 2 & 1.83 \\
\hline \multirow[t]{6}{*}{ NSF } & \multirow[t]{2}{*}{$2 \mathrm{CON}$} & 0.5 & 7 & 8 & 2 & 4 & 2.29 \\
\hline & & 0.7 & 10 & 10 & 2 & 2 & 2 \\
\hline & \multirow[t]{2}{*}{ DELA } & 0.5 & 7 & 21 & 5 & 7 & 6 \\
\hline & & 0.7 & 10 & 21 & 3 & 8 & 4.2 \\
\hline & \multirow[t]{2}{*}{ TREE } & 0.5 & 7 & 6 & 1 & 2 & 1.71 \\
\hline & & 0.7 & 10 & 9 & 1 & 3 & 1.8 \\
\hline
\end{tabular}

than the MILPs. In general the computational times increase as the difficulty increases in the order "MinAug", "MaxCon", and "MaxMCLC". Also, to achieve feasible solutions for these four evaluation metrics, the realization of feasible solutions becomes harder and harder.

\section{SUMMARY AND CONCLUSIONS}

In this paper we have presented a comprehensive treatment of mathematical programming frameworks for the survivable logical topology routing problem in capacitated cross-layer optical networks under multiple cross-layer evaluation metrics. We have enhanced the survivability routing formulation WSRMD given in Section III by developing MILP formulations for (1) minimum logical topology augmentation for guaranteed weakly survivable routing, (2) maximizing the afterfailure connectivity of the logical topology, (3) maximizing the capacity of logical topology, and (4) maximizing the minimum cross-layer cut. An interesting feature of our MILP formulations is that the optimization is carried out in a single stage, in contrast to previous approaches that consider logical
TABLE IV

RESULTS FOR MINIMIZING AUGMENTED LOGICAL EDGES FOR WEAK SURVIVABILITY

\begin{tabular}{|c|c|c|c|c|c|c|}
\hline \multirow{2}{*}{ PhyG } & \multirow{2}{*}{$\log G$} & \multirow{2}{*}{$r_{L / P}$} & \multicolumn{2}{|c|}{ MILP } & \multicolumn{2}{|c|}{ Heuristics } \\
\hline & & & AugEdge & Time (s) & AugEdge & Time (s) \\
\hline \multirow{4}{*}{ DFN } & \multirow{2}{*}{ DELA } & 0.5 & 0 & 0.0696 & 1 & 4E-04 \\
\hline & & 0.7 & 0 & 0.0965 & 0.5 & $6 \mathrm{E}-04$ \\
\hline & \multirow{2}{*}{ TREE } & 0.5 & 2 & 0.2474 & 6 & $3 \mathrm{E}-04$ \\
\hline & & 0.7 & 2.8 & 2.9485 & 6 & $4 \mathrm{E}-04$ \\
\hline \multirow{4}{*}{ E1 } & \multirow{2}{*}{ DELA } & 0.5 & 0 & 0.0535 & 0.9 & $3 \mathrm{E}-04$ \\
\hline & & 0.7 & 0 & 0.0741 & 0.8 & 4E-04 \\
\hline & \multirow{2}{*}{ TREE } & 0.5 & 2 & 0.1341 & 4.2 & $2 \mathrm{E}-04$ \\
\hline & & 0.7 & 2 & 0.4021 & 3.8 & 3E-04 \\
\hline \multirow{4}{*}{ E2 } & \multirow{2}{*}{ DELA } & 0.5 & 0 & 0.3593 & 0.7 & $6 \mathrm{E}-04$ \\
\hline & & 0.7 & 0 & 0.5092 & 1.3 & $8 \mathrm{E}-04$ \\
\hline & \multirow{2}{*}{ TREE } & 0.5 & 2 & 1.5197 & 5.3 & $4 \mathrm{E}-04$ \\
\hline & & 0.7 & 3 & 5.8057 & 8.5 & $6 \mathrm{E}-04$ \\
\hline \multirow{4}{*}{ G3 } & \multirow{2}{*}{ DELA } & 0.5 & 0 & 0.0738 & 0.9 & $3 \mathrm{E}-04$ \\
\hline & & 0.7 & 0 & 0.0934 & 0.6 & $4 \mathrm{E}-04$ \\
\hline & \multirow{2}{*}{ TREE } & 0.5 & 1.4 & 0.1687 & 5 & 2E-04 \\
\hline & & 0.7 & 2 & 0.4438 & 6.3 & $3 \mathrm{E}-04$ \\
\hline \multirow{4}{*}{ G6 } & \multirow{2}{*}{ DELA } & 0.5 & 0 & 0.2942 & 0.6 & 6E-04 \\
\hline & & 0.7 & 0 & 0.3771 & 1.4 & $7 \mathrm{E}-04$ \\
\hline & \multirow{2}{*}{ TREE } & 0.5 & 2 & 1.4556 & 7.1 & $4 \mathrm{E}-04$ \\
\hline & & 0.7 & 2.2 & 27.7924 & 7.1 & $5 \mathrm{E}-04$ \\
\hline \multirow{4}{*}{ NSF } & \multirow{2}{*}{ DELA } & 0.5 & 0 & 0.1293 & 0.4 & $3 \mathrm{E}-04$ \\
\hline & & 0.7 & 0 & 0.1750 & 0.8 & $5 \mathrm{E}-04$ \\
\hline & \multirow{2}{*}{ TREE } & 0.5 & 2 & 0.3120 & 5.6 & $3 \mathrm{E}-04$ \\
\hline & & 0.7 & 2.5 & 13.0718 & 7.2 & $4 \mathrm{E}-04$ \\
\hline
\end{tabular}

subgraphs obtained after each physical link failure. For example, see [7]. The contributions reported in this paper assume considerable significance in view of the increasing interest in network virtualization and topology abstraction incorporating survivability requirements [41][42].

\section{REFERENCES}

[1] T. Lin, Z. Zhou, K. Thulasiraman, G. Xue, and S. Sahni, "Unified mathematical programming frameworks for survivable logical topology routing in IP-over-WDM optical networks," Optical Communications and Networking, IEEE/OSA Journal of, vol. 6, no. 2, pp. 190-203, 2014.

[2] J. Sakaguchi, B. Puttnam, W. Klaus, Y. Awaji, N. Wada, A. Kanno, T. Kawanishi, K. Imamura, H. Inaba, K. Mukasa, R. Sugizaki, T. Kobayashi, and M. Watanabe, "305 Tb/s space division multiplexed transmission using homogeneous 19-Core fiber," Lightwave Technology, Journal of, vol. 31, no. 4, pp. 554-562, Feb. 2013.

[3] T. Lin, Z. Zhou, and K. Thulasiraman, "Logical topology survivability in IP-over-WDM networks: Survivable lightpath routing for maximum logical topology capacity and minimum spare capacity requirements," in Proc. International Workshop on the Design of Reliable Communication Networks (DRCN'11), Oct. 2011, pp. 1-8.

[4] K. Thulasiraman, T. Lin, M. Javed, and G. Xue, "Logical topology augmentation for guaranteed survivability under multiple failures in IPover-WDM optical networks," Optical Switching and Networking, vol. 7, no. 4, pp. 206-214, 2010.

[5] C. Liu and L. Ruan, "A new survivable mapping problem in IP-overWDM networks," IEEE Journal on Selected Areas in Communications, vol. 25 , no. 3, pp. 25-34, 2007.

[6] D. D.-J. Kan, A. Narula-Tam, and E. Modiano, "Lightpath routing and capacity assignment for survivable IP-over-WDM networks," in Proc. International Workshop on Design of Reliable Communication Networks (DRCN'09), Oct. 2009, pp. 37-44. 
TABLE V

COMPUTATION RESULTS WITH 2-CONNECTED PLANAR LOGICAL TOPOLOGIES AND LOGICAL TO PHYSICAL NODE RATIO $=0.5$

\begin{tabular}{|c|c|c|c|c|c|c|c|c|c|c|c|c|c|c|c|c|}
\hline \multirow{3}{*}{ PhyG } & \multicolumn{4}{|c|}{ MinAug } & \multicolumn{4}{|c|}{ МахСара } & \multicolumn{4}{|c|}{ MaxConn } & \multicolumn{4}{|c|}{ MaxMCLC } \\
\hline & \multicolumn{2}{|c|}{ MILP } & \multicolumn{2}{|c|}{ Heuristic } & \multicolumn{2}{|c|}{ MILP } & \multicolumn{2}{|c|}{ Heuristic } & \multicolumn{2}{|c|}{ MILP } & \multicolumn{2}{|c|}{ Heuristic } & \multicolumn{2}{|c|}{ MILP } & \multicolumn{2}{|c|}{ Heuristic } \\
\hline & Obj & Time & Obj & Time & Obj & Time & Obj & Time & Obj & Time & Obj & Time & Obj & Time & Obj & Time \\
\hline$\overline{\text { DFN }}$ & 0.0 & 0.078 & 3.0 & $3 \mathrm{E}-0$ & 3.5 & 2.727 & 1.3 & $6 \mathrm{E}-\mathrm{c}$ & 2 & 0.127 & 1 & $\overline{0.0}$ & 2 & 3.043 & 1 & $\overline{0.003}$ \\
\hline E1 & 0.0 & 0.077 & 4.2 & 2E-04 & 2.7 & 0.984 & 1.2 & $5 \mathrm{E}-04$ & 2 & 0.083 & 1 & 0.002 & 2 & 0.831 & 1 & 0.002 \\
\hline E2 & 0.0 & 1.182 & 4.1 & 4E-04 & 3.3 & 6.184 & 1.3 & 0.001 & 2 & 0.299 & 1 & 0.003 & 2 & 9.268 & 1 & 0.003 \\
\hline G3 & 0.0 & 0.097 & 2.2 & 2E-04 & 5.3 & 0.967 & 1.3 & 5E-04 & 2 & 0.104 & 1 & 0.002 & 2 & 1.681 & 1 & 0.002 \\
\hline G6 & 0.2 & 1.244 & 3.8 & $4 \mathrm{E}-04$ & 3.6 & 5.274 & 1.1 & 9E-04 & 1 & 0.348 & 1 & 0.003 & 2 & 71.62 & 1 & 0.003 \\
\hline NSF & 0.3 & 0.314 & 3.6 & 2E-04 & 3.0 & 1.149 & 0.8 & 7E-04 & 2 & 0.127 & 1 & 0.002 & 2 & 1.177 & 1 & 0.002 \\
\hline
\end{tabular}

TABLE VI

COMPUTATION RESULTS WITH 2-CONNECTED PLANAR LOGICAL TOPOLOGIES AND LOGICAL TO PHYSICAL NODE RATIO $=0.7$

\begin{tabular}{|c|c|c|c|c|c|c|c|c|c|c|c|c|c|c|c|c|}
\hline \multirow{3}{*}{ PhyG } & \multicolumn{4}{|c|}{ MinAug } & \multicolumn{4}{|c|}{ МахСара } & \multicolumn{4}{|c|}{ MaxConn } & \multicolumn{4}{|c|}{ MaxMCLC } \\
\hline & \multicolumn{2}{|c|}{ MILP } & \multicolumn{2}{|c|}{ Heuristic } & \multicolumn{2}{|c|}{ MILP } & \multicolumn{2}{|c|}{ Heuristic } & \multicolumn{2}{|c|}{ MILP } & \multicolumn{2}{|c|}{ Heuristic } & \multicolumn{2}{|c|}{ MILP } & \multicolumn{2}{|c|}{ Heuristic } \\
\hline & Obj & Time & Obj & Time & Obj & Time & Obj & Time & Obj & Time & Obj & Time & Obj & Time & Obj & Time \\
\hline DFN & 0.0 & 0.14 & 3.6 & 3E-04 & 3.5 & 4.325 & 1.2 & $8 \mathrm{E}-04$ & 2 & 0.213 & 1 & 0.003 & 2 & 6.802 & 1 & 0.003 \\
\hline E1 & 0.0 & 0.282 & 2.2 & 3E-04 & 4.0 & 2.184 & 1.3 & $6 \mathrm{E}-04$ & 2 & 0.15 & 1 & 0.002 & 2 & 3.206 & 1 & 0.003 \\
\hline E2 & 0.1 & 185 & 4.7 & $6 \mathrm{E}-04$ & 2.3 & 33.56 & 1.0 & 0.001 & 2 & 4.024 & 1 & 0.004 & 2 & 613.6 & 1 & 0.004 \\
\hline G3 & 0.0 & 0.143 & 6.4 & 3E-04 & 3.7 & 1.247 & 1.4 & 7E-04 & 2 & 0.19 & 1 & 0.002 & 2 & 16.76 & 1 & 0.002 \\
\hline G6 & 0.7 & 8.48 & 5.9 & $5 \mathrm{E}-04$ & 3.3 & 15.3 & 0.8 & 0.001 & 1 & 0.398 & 1 & 0.004 & 1 & 11.66 & 1 & 0.004 \\
\hline NSF & 0.9 & 6.423 & 6.4 & 4E-04 & 4.0 & 3.449 & 0.9 & $8 \mathrm{E}-04$ & 2 & 0.257 & 1 & 0.002 & $\mathrm{xx}$ & $\mathrm{xx}$ & 1 & 0.003 \\
\hline
\end{tabular}

[7] K. Lee and E. Modiano, "Cross-layer survivability in WDM-based networks," in Proc. IEEE INFOCOM'09, Apr. 2009, pp. 1017-1025.

[8] A. Agrawal and R. E. Barlow, "A survey of network reliability and domination theory," Operations Research, vol. 32, pp. 478-492, 1984.

[9] A. Rosenthal and D. Frisque, "Transformations for simplifying network reliability calculations," Networks, vol. 7, pp. 97-111, 1977.

[10] A. Shooman and A. Kershenbaum, "Exact graph-reduction algorithms for network reliability analysis," in IEEE GLOBECOM, Dec. 1991.

[11] _ - "Methods for communicationnetwork reliability analysis: Probabilistic graph reduction," in Annual Reliability and Maintainability Symposium, 1992.

[12] F. Dikbiyik, M. Tornatore, and B. Mukherjee, "Exploiting excess capacity for survivable traffic grooming in optical backbone networks," Optical Communications and Networking, IEEE/OSA Journal of, vol. 6, no. 2, pp. 127-137, Feb. 2014.

[13] R. Karp and M. Luby, "Monte-Carlo algorithms for the planar multiterminal network reliability problem," Journal of Complexity, vol. 1, pp. 45-64, 1985.

[14] D. Karger, "A randomized fully polynomial time approximation scheme for the all terminal reliability problem," SIAM Review, vol. 43, pp. 499 $522,2001$.

[15] C. Colbourn, The Combinatorics of Network Reliability. Oxford University Press, 1987.

[16] S. C. Liew and K. W. Lu, "A framework for characterizing disaster-based network survivability," IEEE J. Selected Areas in Communications, vol. 12, pp. 52-58, 1994.

[17] S. Neumayer, G. Zussman, R. Cohen, and E. Modiano, "Assessing the vulnerability of the fiber infrastructure to disasters," IEEE Trans. Networking, vol. 19, pp. 1610-1623, 2011.

[18] R. M. Karp, Reducibility Among Combinatorial Problems. NY: Plenum Press, 1972, ch. 8, pp. 85-103.

[19] E. Modiano and A. Narula-Tam, "Survivable routing of logical topologies in WDM networks," in Proc. IEEE INFOCOM'01, 2001, pp. 348357.

[20] A. Todimala and B. Ramamurthy, "A scalable approach for survivable virtual topology routing in optical WDM networks," IEEE Journal on Selected Areas in Communications, vol. 25, no. 6, pp. 63-69, Aug. 2007.

[21] J. Strand, A. L. Chiu, and R. Tkach, "Issues for routing in the optical layer," IEEE Communications Magazine, vol. 39, no. 2, pp. 81-87, Feb. 2001.

[22] C. Vadrevu, M. Tornatore, R. Wang, and B. Mukherjee, "Integrated design for backup capacity sharing between IP and wavelength services in IP-over-WDM networks," Optical Communications and Networking, IEEE/OSA Journal of, vol. 4, no. 1, pp. 53-65, Jan. 2012.
[23] M. Kurant and P. Thiran, "Survivable mapping algorithm by ring trimming (SMART) for large IP-over-WDM networks," in Proc. International Conference on Broadband Networks (BROADNETS'04), 2004, pp. 44-53.

[24] S. Lee, J.-C. Liu, and Y. Chen, "An ear-decomposition based approach for survivable routing in WDM networks," in Proc. International Conference on Advanced Information Networking and Applications (AINA'05), vol. 1, Mar. 2005, pp. 459-464.

[25] M. S. Javed, K. Thulasiraman, M. A. Gaines, and G. Xue, "Survivability aware routing of logical topologies: On Thiran-Kurant approach, enhancements and evaluation," in Proc. IEEE GLOBECOM'06, 2006.

[26] M. Javed, K. Thulasiraman, and G. Xue, "Lightpaths routing for single link failure survivability in IP-over-WDM networks," Journal of Communication and Networks, vol. 9, no. 4, pp. 394-401, 2007.

[27] K. Thulasiraman, M. S. Javed, and G. Xue, "Circuits/Cutsets duality and a unified algorithmic framework for survivable logical topology design in IP-over-WDM optical networks," in Proc. IEEE INFOCOM'09, 2009, pp. 1026-1034.

[28] K. Thulasiraman, M. Javed, and G. Xue, "Primal meets dual: A generalized theory of logical topology survivability in IP-over-WDM optical networks," in Proc. International Conference on Communication Systems and Networks (COMSNETS'10), 2010, pp. 128-137.

[29] T. Lin, K. Thulasiraman, and G. Xue, "Robustness of logical topology mapping problems for survivability under multiple physica links failures in IP over WDM optical networks," in COMSNETS 2012, Jan. 2012.

[30] Z. Zhou, T. Lin, K. Thulasiraman, G. Xue, and S. Sahni, "Novel survivable logical topology routing in IP-over-WDM networks by logical protecting spanning tree set," in Ultra Modern Telecommunications and Control Systems and Workshops (ICUMT), $20124^{\text {th }}$ International Congress on, 2012, pp. 650-656.

[31] T. Lin, Z. Zhou, K. Thulasiraman, and G. Xue, "Optimum logical topology routing in an IP-over-WDM optical network and physical link failure localization: An integrated approach," in $5^{\text {th }}$ International Workshop on Reliable Networks Design and Modeling (RNDM'13), 2013.

[32] B. Jaumard and H. A. Hoang, "Design and dimensioning of logical survivable topologies against multiple failures," Optical Communications and Networking, IEEE/OSA Journal of, vol. 5, no. 1, pp. 23-36, Jan. 2013.

[33] C. Colman-Meixner, F. Dikbiyik, M. F. Habib, M. Tornatore, C.-N. Chuah, and B. Mukherjee, "Disaster-survivable cloud-network mapping," Photonic Network Communications, vol. 27, no. 3, pp. 141-153, 2014.

[34] H. Yu, V. Anand, and C. Qiao, "Virtual infrastructure design for surviving physical link failures," The Computer Journal, 2012. 
[35] Q. Deng, G. Sasaki, and C.-F. Su, "Survivable IP over WDM: An efficient mathematical programming problem formulation," in Proc. Allerton Conference on Communication, Control and Computing, Monticello, IL, Oct. 2002

[36] K. Thulasiraman and M. N. S. Swamy, Graphs: Theory and Algorithms. Wiley-Interscience, 1992.

[37] R. K. Ahuja, T. L. Magnanti, and J. B. Orlin, Network Flows: Theory, Algorithms, and Applications. Prentice Hall, Feb. 1993.

[38] K. S. Ho and K. W. Cheung, "Generalized survivable network," IEEE/ACM Transactions on Networking, vol. 15, pp. 750-760, Aug. 2007.

[39] S. Orlowski, M. Pióro, A. Tomaszewski, and R. Wessäly, "SNDlib 1.0-Survivable Network Design Library," in Proc. of $3^{\text {rd }}$ International Network Optimization Conference (INOC), vol. 55, 2007, pp. 278-286.

[40] "Library for efficient modeling and optimization in networks (LEMON)." [Online]. Available: http://lemon.cs.elte.hu/trac/lemon

[41] M. Rahman and R. Boutaba, "Svne: Survivable virtual network embedding algorithms for network virtualization," in Network and Service Management, IEEE Transactions on, vol. 10, no. 2, Jun. 2013, pp. 105118.

[42] R. Ravindran, C. Huang, and K. Thulasiraman, "Topology abstraction service for IP-VPNs," in IEEE Transactions on Parallel and Distributed Systems, vol. 24, Jan. 2013, pp. 184-197.

Zhili Zhou (M'11) received her Ph.D. degree in industrial and system engineering from University of Florida, Gainesville, FL, USA in 2010 and B.S. and M.S. degrees in mathematics from Nanjing University, Nanjing, China in 2003 and 2006, respectively. She is a research staff member at IBM Research Collaboratory, Singapore. Dr. Zhou took second place for the 2011 Pritsker Doctoral Dissertation Award from the Institute of Industrial Engineers for her dissertation. She was the Best Paper Award Runner-up of DRCN'2011 and RNDM'2012. Her research interests are in mixed integer programming, combinatorial optimization, stochastic programming, and quality of service in communication network, transportation network, and power grid. Dr. Zhou is a member of INFORMS.

Tachun Lin (M'08) received the Ph.D. degree in computer science from the University of Oklahoma, Norman, OK, USA, in 2011, and the B.S. and M.S. degrees in computer science from National Chiao Tung University, Hsinchu, Taiwan in 1999 and 2001, respectively. Since 2011, he has been an Assistant Professor of Computing and Technology at Cameron University, Lawton, OK. He was the Best Paper Award Runner-up of DRCN'2011 and RNDM'2012. His research interests include cross-layer network design, network optimization, and graph theory. Dr. Lin is a member of ACM and INFORMS.

Krishnaiyan Thulasiraman (M'72-SM'84-F'90) received his Ph.D. degree from the Indian Institute of Technology (IITM), Madras, India, in 1968. Since 1994 he holds the Hitachi Chair and is Professor with the School of Computer Science, University of Oklahoma, Norman. Prior to joining the University of Oklahoma, he was a Professor (1981-1994) and Chair (1993-1994) of the Electrical and Computer Engineering Department, Concordia University, Montreal, Canada. He has published more than 100 papers in archival journals. He has coauthored with M.N.S Swamy "Graphs, Networks, and Algorithms" (New York: Wiley, 1981) and "Graphs: Theory and Algorithms" (New York: Wiley, 1992) and several book chapters. He has a US patent on Distributed QoS Routing. His research interests have been in graph theory, combinatorial optimization, algorithms and applications in a variety of areas in computer science and electrical engineering, particularly fault diagnosis, tolerance and testing. His most recent interest is in the emerging area of Network Science and Engineering. Dr. Thulasiraman has received several awards and honors: Distinguished Alumnus Award of IIT Madras, IEEE Circuits and Systems Society Technical Achievement Award, IEEE CAS Society Golden Jubilee Medal, Senior Fellow of the Japan Society for Promotion of Science , Gopalakrishnan Endowed Chair Professor of IIT Madras, and Fellow of IEEE, AAAS and the European Academy of Sciences.
Guoliang Xue (M'98-SM'99-F'11) received the Ph.D. degree in computer science from the University of Minnesota, Minneapolis, MN, USA, in 1991. $\mathrm{He}$ is a Professor of computer science and engineering with Arizona State University, Tempe, AZ, USA. His research interests include quality of service provisioning, resource allocation, survivability, security, and privacy issues in networks. He has received Best Paper Awards at IEEE Globecom'2007, IEEE ICC'2011, IEEE MASS'2011, IEEE Globecom'2011, IEEE ICC'2012, and a Best Paper Runner-up at IEEE ICNP'2010. He is an Associate Editor of the IEEE/ACM Transactions on Networking and IEEE Network, and a member of the Standing Committee of IEEE INFOCOM. He is General CoChair of IEEE CNS'2014-IEEE Conference on Communications and Network Security. He was a Keynote Speaker at IEEE LCN'2011, a TPC Co-Chair of IEEE INFOCOM'2010, and a co-Organizer of HC3I'2012-ARO Workshop on Human Centric Computing with Collective Intelligence-Challenges and Research Directions. He served on the editorial boards of Computer Networks, IEEE Transactions on Wireless Communications, and IEEE Transactions on Circuits and Systems. He was a Distinguished Lecturer of the IEEE Communications Society. He is an IEEE Fellow.
Sartaj Sahni (M'78-SM'86-F'88) is a Distinguished Professor of Computer and Information Sciences and Engineering at the University of Florida. He is also a member of the European Academy of Sciences, a Fellow of IEEE, ACM, AAAS, and Minnesota Supercomputer Institute, and a Distinguished Alumnus of the Indian Institute of Technology, Kanpur. In 1997, he was awarded the IEEE Computer Society Taylor L. Booth Education Award "for contributions to Computer Science and Engineering education in the areas of data structures, algorithms, and parallel algorithms", and in 2003, he was awarded the IEEE Computer Society W. Wallace McDowell Award "for contributions to the theory of NP-hard and NP-complete problems." Dr. Sahni was awarded the 2003 ACM Karl Karlstrom Outstanding Educator Award. Dr. Sahni has published over three hundred research papers and written 15 texts. His research publications are on the design and analysis of efficient algorithms, parallel computing, interconnection networks, design automation, and medical algorithms. 